\title{
Imaging features of COVID-19: What we can learn from SARS and MERS (Review)
}

\author{
YUANBIN CHEN $^{1 *}$, LIYA GUO $^{2 *}$, YIQING JIAO $^{2}$, FEITING FAN $^{1}$, JIAN LIU $^{2}$, MING CHEN $^{3}$, \\ HUAIPING YUAN ${ }^{3}$, LIN LIN $^{1}$ and YUGUANG WANG ${ }^{2}$
}

\begin{abstract}
${ }^{1}$ Department of Respiratory Medicine, The Second Clinical College of Guangzhou University of Chinese Medicine, The Second Affiliated Hospital of Guangzhou University of Chinese Medicine and Guangdong Provincial Hospital of Chinese Medicine, Guangzhou, Guangdong 510120;

${ }^{2}$ Department of Respiratory Medicine, Beijing Hospital of Traditional Chinese Medicine, Capital Medical University, Beijing 100010; ${ }^{3}$ Department of Radiology, Fuyang Hospital of Anhui Medical University, Fuyang, Anhui 236000, P.R. China
\end{abstract}

Received June 11, 2020; Accepted December 11, 2020

DOI: 10.3892/etm.2021.9787

\begin{abstract}
Coronavirus disease 2019 (COVID-19) is a highly infectious type of pneumonia caused by severe acute respiratory syndrome coronavirus 2 (SARS-CoV-2) that has rapidly become a global pandemic. COVID-19, SARS and Middle East respiratory syndrome (MERS) are all caused by members of the Coronaviridae family. As expected, emerging genetic and clinical evidence from patients with COVID-19 has indicated that the pathway of infection is similar to that of SARS and MERS. Additionally, much like SARS and MERS, chest imaging serves an important role in the diagnosis, management and follow-up of patients with COVID-19. Although these related viruses present a similar pneumonic pathogenesis, the imaging results have distinguishable features. The current review evaluated the imaging results of patients with SARS and MERS and explored the potential similarities and differences among patients with COVID-19, SARS and MERS at early, progressive, severe and recovery stages, with the aim of improving our understanding of SARS-CoV-2 infections by comparing the features of COVID-19 images with those of SARS and MERS. The current review assessed
\end{abstract}

Correspondence to: Professor Lin Lin, Department of Respiratory Medicine, The Second Clinical College of Guangzhou University of Chinese Medicine, The Second Affiliated Hospital of Guangzhou University of Chinese Medicine and Guangdong Provincial Hospital of Chinese Medicine, 111 Dade Road, Yuexiu, Guangzhou, Guangdong 510120, P.R. China

E-mail: drlinlin620@163.com

Professor Yuguang Wang, Department of Respiratory Medicine, Beijing Hospital of Traditional Chinese Medicine, Capital Medical University, 23 Back Street of Art Gallery, Dongcheng, Beijing 100010, P.R. China

E-mail: wygzhyiaids@126.com

*Contributed equally

Key words: coronavirus disease 2019, pneumonia, viral, radiology whether imaging results had implications for the administration of corticosteroids as treatment for COVID-19. Whether corticosteroids can inhibit inflammatory cytokine storms and reduce the mortality of patients with viral pneumonia remains controversial. However, his review may help radiologists and clinicians to identify viral pneumonia and guide appropriate COVID-19 treatment.

\section{Contents}

1. Introduction

2. Pathogenesis and pathological manifestations of coronavirus

3. Imaging results at different stages of disease progression

4. Suggestions for viral pneumonia chest imaging

5. Imaging implications for corticosteroid therapy

6. Conclusion

\section{Introduction}

The outbreak and subsequent pandemic of Coronavirus disease 2019 (COVID-19) is a public health emergency of international concern (1). As of December 16, 2020, a total of 71,581,532 confirmed cases and 1,618,374 deaths have been reported by the World Health Organization (WHO) (2). COVID-19 is caused by severe acute respiratory syndrome coronavirus 2 (SARS-CoV-2), which was most likely transmitted to humans from wild bats (3). SARS-CoV-2 closely resembles SARS-CoV (79\% sequence identity) and Middle East respiratory syndrome coronavirus (MERS)-CoV (51.8\% identity) (4), both of which are also believed to have originated in bats $(5,6)$. All three of these viruses are members of the Coronaviridae family.

Coronaviruses often cause a series of diseases in humans and animals, ranging from the common cold to more severe illness, such as pneumonia. Zoonotic transmission of coronaviruses, such as SARS-CoV, SARS-CoV-2 and MERS-CoV, may be associated with severe lower respiratory tract infections. These related infections present as pneumonia as the primary clinical feature, sharing symptoms including fever, cough and shortness of breath (7). 
Although viruses within the same family often share similarities in the pathogenesis of pneumonia, their imaging results may exhibit distinguishable features. As for the Coronaviridae family, imaging is an important basis for the diagnosis and evaluation of the underlying viral infections. However, to the best of our knowledge, no studies have summarized the imaging features at different stages of coronavirus pneumonias. To highlight the differences, the current review presents imaging features at the early, progressive, severe and recovery phases of these viruses.

\section{Pathogenesis and pathological manifestations of coro- navirus}

Coronavirus infections first enter susceptible host cells by binding to specific receptors (8). Angiotensin converting enzyme 2 (ACE-2) is a receptor of SARS-CoV and is expressed in tracheobronchial epithelial cells, alveolar epithelial cells and in monocytes and macrophages (9). The downregulation of ACE-2 is considered to be associated with SARS-CoV-induced lung injury (10). The structure of SARS-CoV-2 is similar to that of SARS-CoV, suggesting that the virus may utilize ACE-2 receptors in alveolar type II epithelial cells for cell invasion, thereby replicating into bronchial epithelial cells (11). Smoking and obesity increases the expression of the ACE-2 gene, which explains why smokers and obese individuals are susceptible to infection $(12,13)$. Smoking and obesity are also independent risk factors for the deterioration of COVID-19 infection $(12,13)$. ACE-2 receptors are present in many animals, which enables inter-species contamination (14). The efficiency of binding depends on the affinity between the receptor-binding domain of the virus and the species-specific ACE-2 receptor (14). As such, it is likely that the clinical characteristics and infectivity of SARS and COVID-19 are similar, especially in severe cases (15). Dipeptidyl peptidase-4 is a receptor for MERS-CoV, which is a versatile cell surface protein (16). This virus demonstrates high homology in its primary and tertiary structure with the receptor-binding domain of SARS-CoV (16). However, the simulation of their protein structure exhibits a disparity in the receptors (ACE-2 and dipeptidyl peptidase-4) between the two coronaviruses, and the mechanism that causes this phenomenon remains unclear (17).

Pathological changes of the lung observed in patients with SARS-CoV infection are usually diffuse, involving several lung lobes and manifesting as diffuse alveolar damage $(18,19)$. Histopathological assessment of MERS-CoV infection has indicated necrotizing pneumonia, pulmonary diffuse alveolar damage and acute kidney injury (20). On 27 January 2020, a death attributed to COVID-19 was pathologically dissected for the first time in China. The pulmonary manifestations were diffuse alveolar injury and hyaline membranes, which are consistent with acute respiratory distress syndrome (ARDS) (21). However, another report of five cases revealed that no viral cytopathic changes were observed in COVID-19. Moreover, diffuse alveolar injury with hyaline membrane formation, inflammation and type II pneumocyte hyperplasia were not prominent (22). Therefore, microvascular injury alongside thrombosis may serve an important role when hyaline membrane formation is not prominent in certain patients. Although the overall pathological manifestations of the lungs are similar to SARS and MERS, there are also differences.

\section{Imaging at different stages of disease progression}

COVID-19, SARS and MERS are novel infectious diseases with general stages of progression that are consistent with other infectious diseases, such as influenza. These can manifest as different clinical types following the natural course of the disease and during the pathophysiological changes that occur $(18,19,23)$. Combined with clinical classification and imaging features $(18,23)$, the progression of these diseases is currently classed into four stages: Early, progressive, severe and recovery.

Early stage. In this stage, clinical symptoms exhibited by patients with COVID-19 are mild to moderate, although some patients are asymptomatic. Usually there is no imaging evidence of pneumonia in patients that are asymptomatic or those with mild symptoms, and the changes of imaging are often atypical, which may result in omissions. For example, Zhang et al (24) demonstrated that high-resolution computed tomography (HRCT) exhibited multiple instances of ground-glass opacity (GGO) and may be accompanied by consolidation in patients with early stage COVID-19. The study also revealed that certain patients did not present imaging results that were indicative of pneumonia, and others exhibited normal chest radiographs, but HRCT results revealed pneumonia. Therefore, with imaging as an important supplement to the screening of COVID-19, HRCT should be recommended as the initial imaging technique, as X-rays often result in missed diagnoses in the early stage. Pulmonary CT manifestations are usually as follows: i) GGO or consolidation changes, in which multiple lesions on the bilateral lung are common. The scope of consolidation is small and localized (25); ii) the density of the lesions is uneven, and they are distributed in a localized manner. Generally, only parts of the lung segment are affected, mostly within the extrapulmonary zone and the lower lung (25); iii) there are no manifestations of mediastinal or hilar lymphadenopathy, pleural thickening or pleural effusion (26). Typical HRCT patterns of patients with early stage COVID-19 are presented in Fig. 1A and B.

SARS is an acute infectious disease with fever as the first and primary symptom, often occurring without upper catarrhal symptoms (18). At the early stage, the time from clinical symptom presentation to chest imaging abnormalities is generally only 2-3 days. X-rays and CT scans of the lungs demonstrate small or round-shaped GGO, with some patients presenting this alongside lung consolidation. Single lesions are more common, and those involving the lung segment are rare. Most of the lesions are distributed in the lower field and lateral bands of both lungs (18).

The early stage of MERS usually manifests as an acute respiratory infection. Patients with low immune function or underlying diseases, including coronary heart disease and diabetes, may have more severe symptoms, such as dyspnea (27). However, for those without underlying disease, symptoms are mild or asymptomatic, and some patients do not exhibit imaging changes (28). The primary features of the lung that are visible in HRCT images are GGO changes 

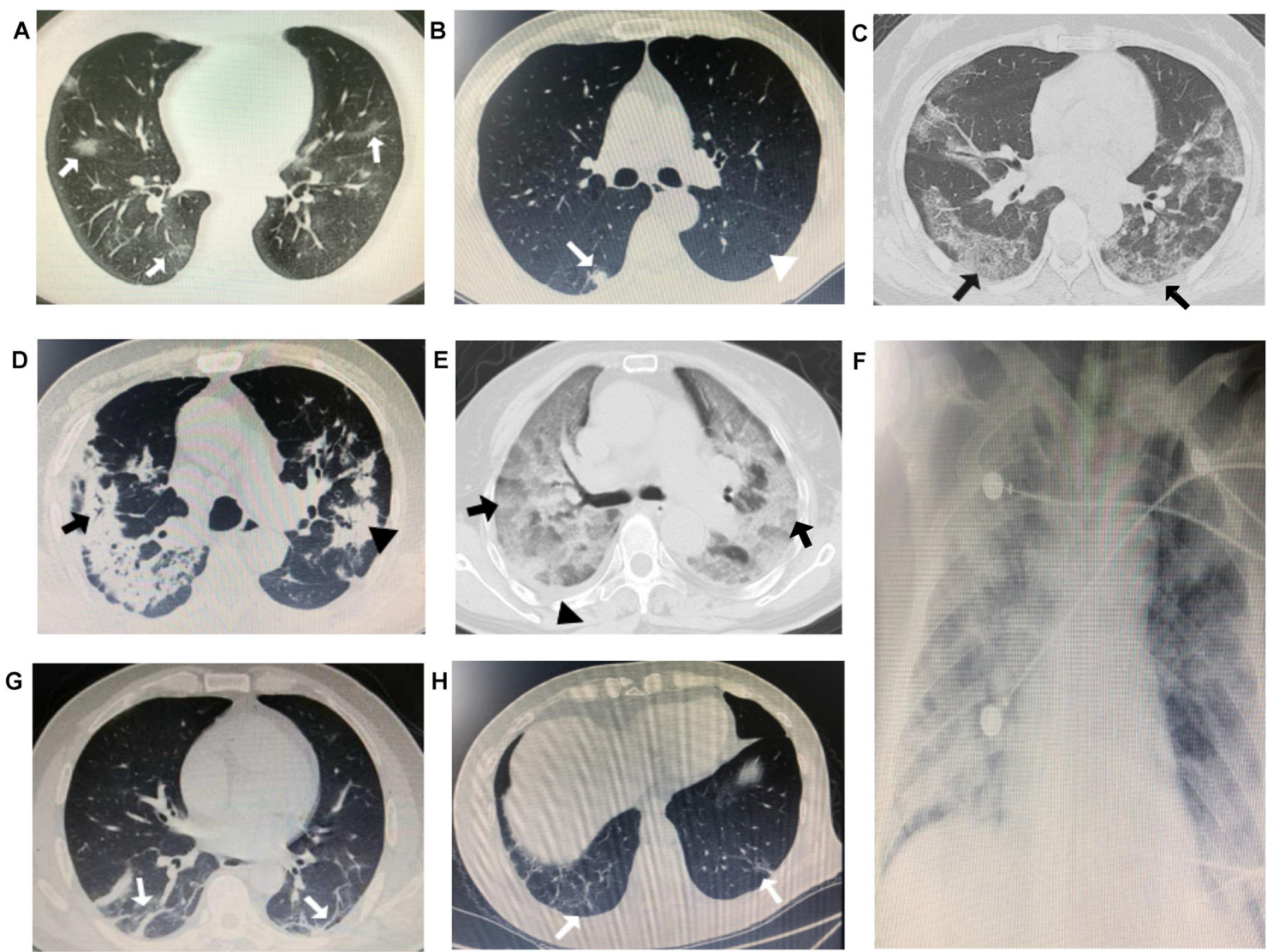

Figure 1. Imaging patterns of COVID-19. (A) A 29-year-old male with early stage COVID-19 exhibited mild fever, aversion to cold, dry cough and dizziness. Chest CT demonstrated multiple localized light and thin GGOs in the bilateral lower lung (white arrows). (B) A 61-year-old male with early stage COVID-19 presented fever and limb weakness. Chest CT indicated localized round consolidation of the right lower lung (white arrow) and centrilobular emphysema of the left lower lung with reduced density and no visible wall was visible in the bilateral lung (white arrowhead). (C) A 47-year-old female with progressive stage COVID-19 presented with fever, cough and shortness of breath. Chest CT revealed multiple GGOs in the subpleural lung, accompanied by reticular changes, presenting as 'crazy-paving pattern' (black arrows). (D) A 71-year-old male with progressive stage COVID-19 exhibited wheezing, a cough and chest pain. Chest CT indicated multiple consolidations in the bilateral lung (black arrowhead), and signs of air bronchogram (black arrow). (E) A 72-year-old female with severe stage COVID-19 presented with fever, dyspnea, weakness and fatigue. Chest CT demonstrated large and diffuse GGO with mixed consolidation in the bilateral lung, presenting as 'white lung' coalesced with a thickened interlobular septum (black arrows) and a small degree of pleural effusion in the right lung (black arrowhead). (F) A 35-year-old woman with severe stage COVID-19 exhibited dyspnea and a minimally productive cough. X-rays revealed an extensive range of lesions, with diffuse and exudative lesions in the bilateral lungs. (G) A 56-year-old male with recovery stage of COVID-19 presented with a dry cough only. Chest CT revealed pulmonary fibrosis, scaring and stripe shadows in the bilateral lower lung (white arrows). (H) A 71-year-old male with recovery stage COVID-19 presented with a mild cough. Chest CT indicated pulmonary fibrosis and stripe shadows in the bilateral lower lung (white arrows). COVID-19, Coronavirus disease 2019.

and occasionally mixed consolidation or small nodules, most of which are distributed in the subpleural and basal lung regions $(29,30)$. Some cases may demonstrate varying degrees of pleural effusion $(31,32)$.

Early imaging features of the three diseases share several similarities. GGO is the primary symptom, and a small degree of consolidation may be observed. Lesions generally do not affect the entire lung segment and are most common in the lower lung field and lateral bands. However, certain patients with COVID-19 do not present changes in chest images in the early stage, whereas patients with SARS demonstrate pneumonia within a short period of time (2-3 days). The reasons for this difference may be the duration of the viral incubation period, the method of virus detection used or the popularity of pulmonary HRCT. The reason of pulmonary HRCT being not commonly used is attributed to the cost of the CT examination and the doctors' cognitive level of the characteristics of different coronavirus diseases. The aforementioned similarities and differences of early-stage features of COVID-19, SARS and MERS are summarized in Tables I and II.
Progressive stage. There are several pulmonary HRCT imaging features of COVID-19: i) The confluence or expansion of GGO lesions may be demonstrated, with some being accompanied by certain reticular changes, such as the 'crazy-paving pattern'. Sometimes lesions appear as consolidations, and signs of air bronchogram may be observed. GGO can also appear around consolidations or other lung fields (26). ii) The lesion area may increase due to multiple lesions fusing together or through diffusion into multiple lung lobes, demonstrating asymmetric distribution in the lungs. This is most commonly observed feature in the middle and lateral bands. iii) Enlargement of the mediastinum and hilar lymph nodes may occur, although this is rare. The lesions progress rapidly and clear changes in imaging morphology appears within a short period (several days) $(25,26)$. Active treatment is required and the possibility of ARDS must be considered (26). Typical imaging patterns of progressive stage COVID-19 are presented in Fig. 1C and D.

In the progressive stage of SARS, fever and other symptoms of infection persist, with imaging demonstrating progressive 
Table I. Similarities in imaging features of COVID-19, SARS and MERS at each stage.

\begin{tabular}{|c|c|}
\hline Stage & Similarities between COVID-19, SARS and MERS \\
\hline Early & $\begin{array}{l}\text { GGO is the primary feature. A small degree of consolidation is visible. Lesions are typically } \\
\text { localized and mostly involve the dorsal or lateral segments of the middle and lower part of the lungs. } \\
\text { Lesions are more concentrated in the bilateral lower lung and the extrapulmonary band. }\end{array}$ \\
\hline Progressive & $\begin{array}{l}\text { The scope of GGO is enlarged and there is a high density of consolidation lesions. GGO may also be } \\
\text { combined with consolidation. The scope of the lesion is more extensive, involving the bilateral lung or } \\
\text { multiple lung fields. The disease progresses rapidly, with white lung being visible due to } \\
\text { the deterioration of the infection. The possibility of ARDS should be considered. }\end{array}$ \\
\hline Severe & $\begin{array}{l}\text { Diffuse lesions are present in the bilateral lung. The changes in the images are observed over a short } \\
\text { period of time and signs of white lung may appear, indicating that ARDS has developed. }\end{array}$ \\
\hline Recovery & $\begin{array}{l}\text { The scope and density of the lesions subside or disappear. Pulmonary fibrosis persists in some patients. } \\
\text { Imaging lesions usually disappear after the improvement of clinical symptoms. }\end{array}$ \\
\hline
\end{tabular}

COVID-19, Coronavirus disease 2019; SARS, severe acute respiratory syndrome; MERS, Middle East respiratory syndrome; GGO, ground-glass opacity; ARDS, acute respiratory distress syndrome.

Table II. Differences in imaging features of COVID-19, SARS and MERS at each stage.

\begin{tabular}{|c|c|c|c|}
\hline Stage & Imaging features COVID-19 & SARS & MERS \\
\hline Early & $\begin{array}{l}\text { No evidence of pneumonia is } \\
\text { demonstrated in images obtained } \\
\text { from patients with mild disease. } \\
\text { Multiple lesions on the bilateral } \\
\text { lung are common. Pleural effusion } \\
\text { is rare. X-rays have a high rate } \\
\text { of misdiagnoses, and HRCT }\end{array}$ & $\begin{array}{l}\text { Pneumonia associated } \\
\text { imaging changes are common. } \\
\text { Single lesions are more } \\
\text { common on the unilateral lung. } \\
\text { Pleural effusion is rare. } \\
\text { X-rays or HRCT } \\
\text { are recommended. }\end{array}$ & $\begin{array}{l}\text { There is no evidence of pneumonia } \\
\text { on the images of certain patients } \\
\text { with mild or asymptomatic disease. } \\
\text { Single or multiple lesions are visible } \\
\text { on the unilateral or bilateral lung. } \\
\text { Easily coalesces with pleural effusion. } \\
\text { X-rays or HRCT are recommended. }\end{array}$ \\
\hline
\end{tabular}

Progressive There are signs of air bronchogram in consolidation lesions. There is usually no pleural effusion.

Severe

The time-point of developing severe disease after onset isuncertain.

Primarily manifests with

consolidation lesions in combination with GGO. There is a small degree of pleural effusion in certain patients.

Recovery Mostly occurs within 1-2 weeks after onset. The condition can be further aggravated with increased or new lesions.
Signs of air bronchogram or GGO halos are rare. Pleural effusion is also rare.

Mostly occurs within 2-3 weeks after onset. Primarily manifests with GGO in combination with consolidation lesions. There is a small degree of pleural effusion.

Mostly occurs within 2-3 weeks after onset. The condition is relatively stable, and recurrence is unusual.
GGO halos are visible.

Easily coalesces by varying degrees with pleural effusion.

Mostly occurs within

1 week after onset.

Primarily manifests

with GGO in combination

with consolidation lesions.

Pleural effusions of varying

degrees are more common.

Mostly occurs within

2 weeks after

onset. The condition

is relatively stable, and

recurrence is unusual.

COVID-19, Coronavirus disease 2019; SARS, severe acute respiratory syndrome; MERS, Middle East respiratory syndrome; HRCT, high-resolution computed tomography; GGO, ground-glass opacity.

deterioration within 3-7 days after onset (18). The features of pulmonary CT imaging are as follows: i) GGO increases or occurs alongside consolidation, and the lesions are large or diffuse; and ii) the lesions may spread from one lung field to multiple lung fields, with lesions of the unilateral lung progressing into the bilateral lung. Lesions are distributed in 
multiple lung lobes, but primarily in the lower lobe, with a mixed distribution in the inner and outer lung fields $(18,33)$. However, central distributions are rare (18). At this stage, pulmonary lesions proliferate.

From the date of onset, MERS can progress within 2-3 weeks. Additionally, certain patients may progress rapidly from asymptomatic infection to pneumonia within 4-7 days (34-36), demonstrating pneumonia-associated clinical symptoms and typical imaging changes (37). Multifocal nodular consolidation with rapid progression in the lower lung and the lateral zone of the lung may be demonstrated in pulmonary HRCT images. This is often accompanied with a GGO halo, mixed consolidation (34) and bilateral interstitial infiltration (38).

Overall, the presentation of COVID-19, SARS and MERS is similar in the progressive stage. Each demonstrates larger lesion areas, pronounced consolidation shadows and a wide distribution of the lesions. During this stage, the disease progresses rapidly and can lead to ARDS if the condition worsens. The differences observed between infections include the presentation of pleural effusions, evidence of an air bronchogram or evidence of a GGO halo. The similarities and differences in images at the progressive stage of COVID-19, SARS and MERS are summarized in Tables I and II.

Severe stage. A retrospective study by Guan et al (39) summarized the clinical characteristics of 1,099 patients with COVID-19 in 552 hospitals located in China. The results revealed that $15.7 \%$ of patients developed severe pneumonia. An additional study demonstrated this value to be $25.5 \%$ (40). For COVID-19 to be classified as severe, patients must meet any of the following criteria: i) Respiratory distress (respiratory rate, $\geq 30$ breaths'min), ii) oxygenation index $\leq 300 \mathrm{mmHg}$, iii) finger oxygen saturation $\leq 93 \%$ in a resting state and iv) chest images presenting $>50 \%$ lesion progression within 24-48 h (23).

Pulmonary HRCT images suggest that as the GGO density increases, the lesions fuse and progress into multiple, large and diffuse consolidations on the bilateral lung from the periphery to the center, involving multiple lobes and presenting as "white lung". Additionally, certain patients demonstrate a small degree of pleural effusion. This phase of treatment is difficult, and the mortality rate is $49 \%$. Certain patients may exhibit insignificant changes in imaging, despite worsening clinical symptoms. This is most common in patients with other underlying diseases, such as cerebral vascular disease (26). Typical imaging patterns of patients with severe COVID-19 are presented in Fig. 1E and F.

The majority of patients with SARS enter the very severe stage 2-3 weeks after onset. Imaging morphology and lesion range change rapidly at this stage, with some changes in chest imaging occurring within 1-3 days $(33,41)$. Patients may demonstrate 'white lung' in images, which indicates that ARDS had occurred (41). ARDS may develop in 10-15\% of patients with SARS $(41,42)$, which is a life-threatening condition. The presentation of 'white lung' in images may indicate poor prognosis and death, but it can also disappear after treatment in certain patients (18). In addition, SARS in the severe stage is prone to relapse. The images of certain patients may indicate that the lesion has disappeared; however, it may then reappear or become aggravated in a short period of time (18).

Most severe cases of MERS progress into severe pneumonia within 1 week. This can lead to ARDS, acute renal failure, septic shock or multiple organ failure. Patients with MERS are more prone to acute renal failure than those with SARS $(29,37)$. The WHO reported that $12.4 \%$ of patients with MERS develop ARDS (43). The primary imaging feature of this stage is bilateral interstitial infiltration that progresses rapidly (44). Furthermore, imaging typically indicates the deterioration of lesions, including those patients previously presenting with 'white lung'. The changes in images are rapid and require attentive monitoring (30).

The differences in imaging features between COVID-19, SARS and MERS include the progression rate of lesions, the likelihood of pleural effusion, the main clinical manifestations of consolidation or GGO and whether the consolidation or GGO is the primary manifestation. The similarities and differences in images at the severe stage of these diseases are summarized in Tables I and II.

Recovery stage. The recovery stage of COVID-19 typically occurs 1-2 weeks after the onset of pneumonia. The imaging features include a decrease in the scope and density of lesions, a gradual disappearance in consolidation lesions and the beginning of organizing pneumonia. The lesions may completely disappear, or part of the funicular shadow may remain $(25,26)$. Changes in imaging at the recovery stage generally lag behind the improvement of clinical symptoms (25). However, the lesions may subsequently enlarge, or new lesions may appear in certain cases (25). Typical imaging patterns of patients in recovery stage of COVID-19 are presented in Fig. $1 \mathrm{G}$ and $\mathrm{H}$.

The majority of SARS cases proceed to the recovery stage within 2-3 weeks after onset. The range and density of the lesions observed in images may exhibit a gradual decrease, or they may disappear entirely. Pulmonary fibrosis is also a common imaging feature during recovery (18). Patients with severe cases are more prone to pulmonary fibrosis compared with those with ordinary infection, with fibrosis disappearing at a slower rate (45). The majority of patients recover within 2-3 months post-discharge (18) and 7-8\% of patients demonstrate pronounced sequelae of pulmonary fibrosis (46).

The imaging features of patients with MERS during the recovery stage include the scope of lesions decreasing significantly and certain patients experiencing left pulmonary fibrosis. The rate of improvement in clinical symptoms is slightly faster than what is exhibited by images $(30,34)$. A case study reporting imaging follow-up revealed that abnormalities in multiple nodules combined with GGO declined after treatment, but progression in fibrosis was observed (34).

The overall condition of patients with COVID-19, SARS and MERS during the recovery stage tends to be stable, and images usually indicate that the lesions have gradually disappeared. In general, changes in imaging occur later than improvements in clinical manifestations. Regarding disease progression, the recovery stage of COVID-19 is earlier than that of SARS; however, some patients with COVID-19 may exhibit recurrent conditions that require attention (47). The similarities 


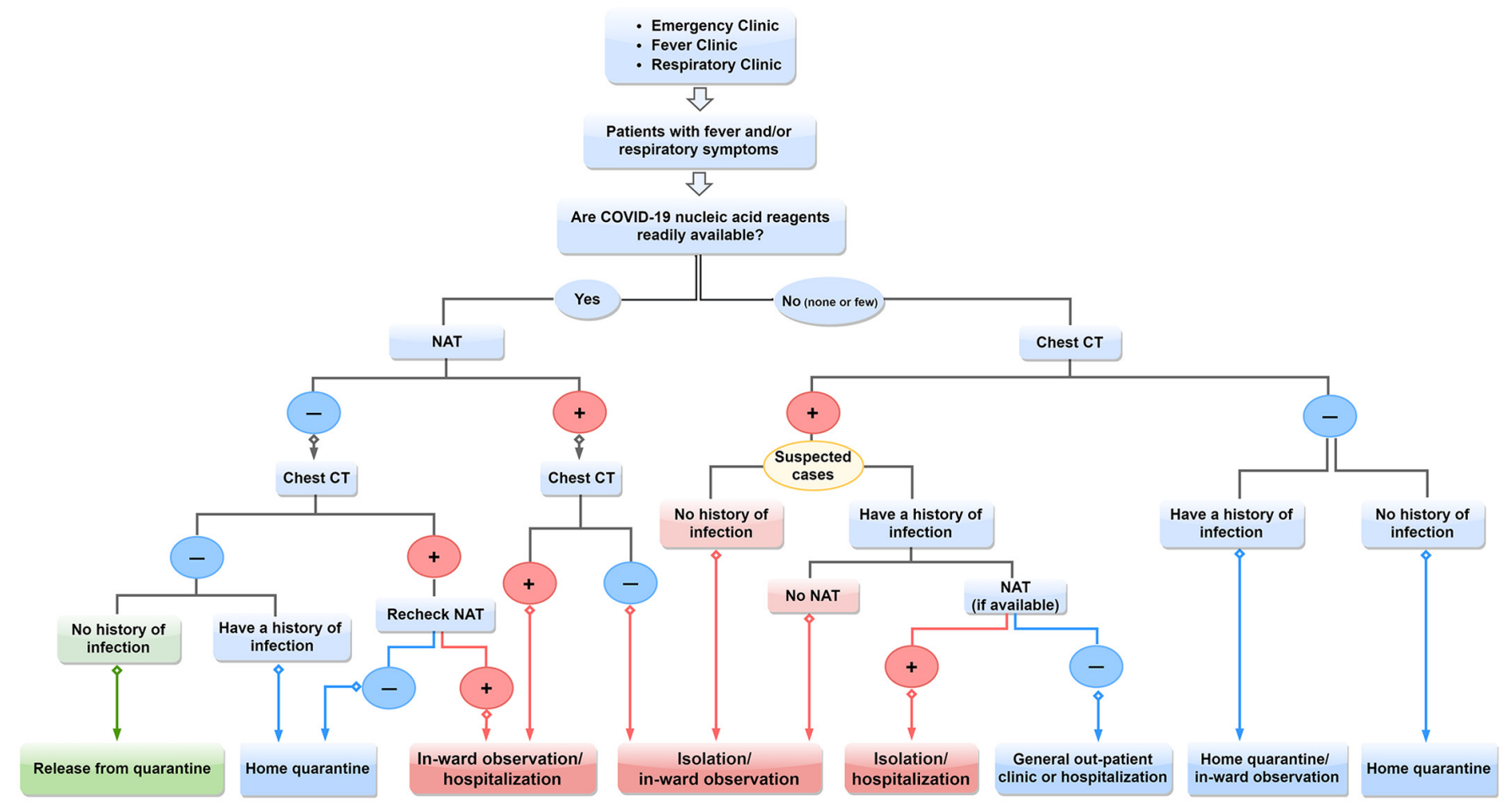

Figure 2. Screening flowchart of COVID-19. -, negative; +, positive; COVID-19, Coronavirus disease 2019; NAT, nucleic acid test.

and differences in images at the recovery stage of COVID-19, SARS and MERS are summarized in Tables I and II

\section{Suggestions for viral pneumonia chest imaging}

Chest imaging is important to the diagnosis, management and prognosis of patients with viral pneumonia. The benefits of imaging are numerous.

Evaluation of imaging when diagnosing patients at their first hospital visit. The majority of chest images obtained at the first hospital visit in patients with SARS or MERS are abnormal, which aids the successful and early diagnosis of patients $(18,31)$. However, for patients exhibiting early stage COVID-19, the interpretation of imaging results requires careful attention. Guan et al (39) demonstrated that radiologic abnormalities were not identified in the initial presentation of 17.9 and $2.9 \%$ of non-severe and severe cases, respectively. Although the detection of viral nucleic acid is the first method used to diagnose COVID-19, and while chest imaging should not replace this method, many countries are still facing a shortage of nucleic acid reagents, particularly in poor and developing countries $(48,49)$. During the early stages of COVID-19 outbreaks or during large-scale outbreaks, the flowchart for screening COVID-19 presented in Fig. 2, which was constructed based on our previous clinical experience in Wuhan, may be used as a reference for diagnosis in the absence of nucleic acid testing kits.

Evaluation of disease progression and prognosis by imaging. A previous study has indicated that consolidation lesions could serve as a marker of disease progression or a more severe disease state following COVID-19 infection (50).
Furthermore, the consolidation of lesions can be indicative of disease progression or deterioration (51). A second study also indicated that pleural effusion was identified in $33 \%$ of patients with MERS and was associated with poor prognosis (52). An observational study on the chest radiographs of 55 patients with MERS revealed higher rates of pneumothorax and pleural effusion in deceased patients compared with those who had recovered (53).

Evaluation of lesion scope for prognosis. A previous study that assessed the clinical outcome of 70 patients with MERS revealed that the imaging manifestations of the bilateral lung were a risk factor for intensive care unit admission (44). Imaging manifestations of patients with severe COVID-19 include bilateral lung involvement and interstitial change, which indicates poor prognosis (39). Patients with SARS may demonstrate multiple lung lobe lesions. If the range of lesions usually exceeds one third of the lung lobe, the patient may be at the severe stage of infection (54).

Evaluation of disease progression speed for prognosis. Two consecutive contrast HRCT scans of the lungs that demonstrate rapid lesion progression, mainly consisting of consolidation combined with GGO and air bronchogram, may indicate that the patient is at a high risk of COVID-19 progression from a common type to severe type (55). X-rays obtained in a previous study demonstrated progression in $>50 \%$ of lesions within 48 $\mathrm{h}$, which was indicative of severe SARS (54).

Effect of early diagnosis on prognosis. As of December 16, 2020, the mortality rate of patients with COVID-19 has been reported to be $2.26 \%$ worldwide (2). However, among male patients aged $\geq 60$, an initial diagnosis of severe pneumonia 
and a delay in diagnosis were associated with elevated mortality rates (40). Early diagnosis is an important measure for the prevention of severe pneumonia or death, and imaging examination may therefore be helpful. The majority of patients with severe cases demonstrate imaging abnormalities at the time of onset, and consolidation generally indicates disease progression. Pleural effusion, pneumothorax, bilateral lung involvement and the rapid progression of lesions can be indicative of severe cases. If necessary, chest X-rays or pulmonary CT scans should be re-examined within $48 \mathrm{~h}(25,56)$. Chest imaging can be used for early diagnosis, the early identification of severe cases and for early treatment guidance. It can also reduce the risk of death (25).

\section{Imaging implications for corticosteroid therapy}

The current COVID-19 pandemic has urged the scientific community internationally to find methods in terms of therapeutics and vaccines to control SARS-CoV-2. Despite the rapidly increasing volume of scientific data on the possible treatments of COVID-19, none have yet demonstrated unequivocal clinical utility against the virus (57). For COVID-19, the immunization of a population through vaccination is recognized as a public health priority (58). WHO and other national organizations collaborate on the response and tracking of the COVID-19 pandemic, advising on critical interventions and attempting to develop safe and effective vaccines (2). As of 12 December 2020, three COVID-19 vaccines (Pfizer, Moderna and AstraZeneca) have been authorized by certain national regulatory authorities. None have yet received WHO emergency use listing' prequalification authorization, but an assessment of the Pfizer vaccine by the end of December and of other candidates soon thereafter is expected by WHO (59).

As research and clinical trials continue to develop vaccines and therapies, scientists have gained an increased understanding of Coronaviridae characteristics. For example, the acute aggravation of SARS and MERS is considered to be associated with cytokine storms. Previous studies have suggested that prolonged and dysregulated cytokine production occurs in SARS (60), and large increases in pro-inflammatory cytokines in the serum of patients with SARS have been associated with extensive inflammatory damage to the lungs (61). Additionally, Mahallawi et al (62) analyzed cytokine responses in plasma samples obtained from patients with MERS. The results demonstrated a marked pro-inflammatory cytokine response during the acute phase of MERS-CoV infection. Furthermore, Liu et al (63) suggested that a cytokine storm may also be associated with disease severity and should be considered as an important cause of death in patients with severe and critical COVID-19.

Corticosteroids are commonly used to treat patients with severe pneumonia, with the purpose of inhibiting abnormal pathological immune responses and reducing systemic inflammation. Chest imaging evaluation may also provide a basis for assessing the severity of lung injury to guide the use of corticosteroids (64). The evaluation of lung images may help to determine whether corticosteroids can be used in patients with SARS. The imaging features of corticosteroid use correspond to an X-ray exhibiting large or multiple pulmonary shadows that progress rapidly, and a lesion area that increases $>50 \%$ within $48 \mathrm{~h}$ and accounts for over one quarter of the bilateral lung area. However, previous studies have demonstrated that corticosteroids may increase the mortality rate of patients with SARS and delay viral clearance $(65,66)$. At present, there are conflicting opinions on whether to administer corticosteroids to patients with MERS. The Chinese expert consensus recommendation for the use of corticosteroids for COVID-19 suggests that imaging-confirmed pneumonia and rapid progression are conditions for which corticosteroid application must be considered (67). According to China's Novel Coronavirus Pneumonia Diagnosis and Treatment Plan (Trial Version 7) (3), patients with progressive deterioration of the oxygenation index, rapid progression that is visible following imaging and patients exhibiting an increased inflammatory response may receive a short course of corticosteroids for 3-5 days as appropriate. An early short course of methylprednisolone in hospitalized patients with moderate to severe COVID-19 has been confirmed to reduce escalation of care and length of hospital stay (68).

It is important to avoid high-dose corticosteroid shock therapy, as this approach delays the clearance of coronavirus due to immunosuppression (69). The dosage and course of treatment should be adjusted based on the severity of the patient's condition and disease status, with an overall goal of medium dosage and short course of treatment (67,70). For example, methylprednisolone is usually administered at a dosage of 40-160 mg once per day for 5 days, with a maximum course lasting no more than 7-10 days (70).

Whether corticosteroids can prevent inflammatory cytokine storms and reduce the mortality of patients with viral pneumonia remains unclear. It is expected that high-quality clinical studies (large sample, multicenter, randomized, double-blind, placebo-controlled trials) will provide more evidence to guide practice. Once corticosteroid therapy is required, chest imaging evidence is a crucial factor to consider.

\section{Conclusion}

Although the imaging results of COVID-19, SARS and MERS demonstrate clear similarities, there are also differences that must be considered. The present review has summarized the key imaging features of coronavirus pneumonia at different stages in order to aid its diagnosis. The imaging features of SARS and MERS provide a reference for the better prevention and control of COVID-19.

\section{Acknowledgements}

Not applicable.

\section{Funding}

The current study was supported by The Guangdong Provincial Key Laboratory of Research on Emergency in TCM (grant no. 2017B030314176).

\section{Availability of data and materials}

Not applicable. 


\section{Authors' contributions}

LL and YGW conceived the project. YBC and LYG contributed to draft the manuscript. YQJ, FTF and JL contributed to searching the electronic databases and interpreting the image data. LL, YGW, MC and HPY revised the contents and polished the language of the translation. All authors read and approved the final manuscript.

\section{Ethics approval and consent to participate}

The images in the manuscript came from a clinical study of Chinese medicine in the treatment of COVID-19, which was approved by the Ethics Committee of Guangdong Provincial Hospital of Chinese medicine (Guangzhou, China; approval no. 2020-049-01). All participants provided informed consent for participation in the study. In the informed consent form, it was clearly stated that the participant agreed that their data and biological specimens of this project may be used for other studies.

\section{Patient consent for publication}

Not applicable.

\section{Competing interests}

The authors declare they have no competing interests.

\section{References}

1. World Health Organization: Clinical Management of Severe Acute Respiratory Infection when Novel Coronavirus (nCoV) Infection is Suspected: Interim Guidance. January 11, 2020.

2. WorldHealth Organization: CoronavirusDisease 2020 (COVID-19) Pandemic. 16 December, 2020. Available from: https://www.who. int/emergencies/diseases/novel-coronavirus-2019.

3. National Health Commission of the People's Republic of China: China's Novel Coronavirus Pneumonia Diagnosis and Treatment Plan (Trial version 7). March 3, 2020. Available from: http:/ www.nhc.gov.cn/yzygj/s7653p/202003/46c9294a7dfe4cef80dc7 f5912eb1989.shtml.

4. Ren LL, Wang YM, Wu ZQ, Xiang ZC, Guo L, Xu T, Jiang YZ, Xiong Y, Li YJ, Li XW, et al: Identification of a novel coronavirus causing severe pneumonia in human: A descriptive study. Chin Med J (Engl) 133: 1015-1024, 2020.

5. Fan Y, Zhao K, Shi ZL and Zhou P: Bat Coronaviruses in China. Viruses 11: 210, 2019.

6. Docea AO, Tsatsakis A, Albulescu D, Cristea O, Zlatian O, Vinceti M, Moschos SA, Tsoukalas D, Goumenou M, Drakoulis N, et al: A new threat from an old enemy: Re emergence of coronavirus (Review). Int J Mol Med 45: 1631-1643, 2020.

7. YeZW, Yuan SF, Yuen KS, Fung SY, Chan CP and Jin DY: Zoonotic origins of human coronaviruses. Int J Biol Sci 16: 1686-1697, 2020

8. Huang C, Wang Y, Li X, Ren L, Zhao J, Hu Y, Zhang L, Fan G, Xu J, Gu X, et al: Clinical features of patients in-fected with 2019 novel coronavirus in Wuhan, China. Lancet 395: 497-506, 2020.

9. Bourgonje AR, Abdulle AE, Timens W, Hillebrands JL, Navis GJ, Gordijn SJ, Bolling MC, Dijkstra G, Voors AA, Osterhaus AD, et al: Angiotensin-converting enzyme 2 (ACE2), SARS-CoV-2 and the pathophysiology of corona-virus disease 2019 (COVID-19). J Pathol 251: 228-248, 2020.

10. Kuba K, Imai Y, Rao S, Gao H, Guo F, Guan B, Huan Y, Yang P, Zhang Y, Deng W, et al: A crucial role of angi-otensin converting enzyme 2 (ACE2) in SARS coronavirus-induced lung injury. Nat Med 11: 875-879, 2005.

11. Rabaan AA, Al-Ahmed SH, Haque S, Sah R, Tiwari R, Malik YS, Dhama K, Yatoo MI, Bonilla-Aldana DK and Rodriguez-Morales AJ: SARS-CoV-2, SARS-CoV, and MERS-COV: A comparative overview. Infez Med 28: 174-184, 2020.
12. Goumenou M, Sarigiannis D, Tsatsakis A, Anesti O, Docea AO, Petrakis D, Tsoukalas D, Kostoff R, Rakitskii V, Spandidos DA, et al: COVID 19 in Northern Italy: An integrative overview of factors possibly influencing the sharp increase of the outbreak (Review). Mol Med Rep 22: 20-32, 2020.

13. Petrakis D, Margină D, Tsarouhas K, Tekos F, Stan M, Nikitovic D, Kouretas D, Spandidos DA and Tsatsakis A: Obesity a risk factor for increased COVID 19 prevalence, severity and lethality (Review). Mol Med Rep 22: 9-19, 2020.

14. Goumenou M, Spandidos DA and Tsatsakis A: [Editorial] Possibility of transmission through dogs being a con-tributing factor to the extreme Covid 19 outbreak in North Italy. Mol Med Rep 21: 2293-2295, 2020.

15. Zhou P, Yang X, Wang X, Hu B, Zhang L, Zhang W, Si HR, Zhu Y, Li B, Huang CL, et al: Discovery of a novel coronavirus associated with the recent pneumonia outbreak in humans and its potential bat origin. Nature: Jan 23, 2020 (Epub ahead of print). doi: $10.1038 / \mathrm{s} 41586-020-2012-7$

16. Meyerholz DK, Lambertz AM and McCray PB Jr: Dipeptidyl peptidase 4 distribution in the human respiratory tract: Implications for the Middle East respiratory syndrome. Am J Pathol 186: 78-86, 2016.

17. Tao JL, Wang AX, Guan CL, et al: Protein-protein docking research on spike protein of three human-coronavirus and its receptors. J Logis Univ CPAPF 26: 392-396, 2017.

18. Chinese Medical Association, China Association of Chinese Medicine: Diagnosis and treatment of severe acute respiratory syndrome (SARS). Chin Med J (Engl) 83: 1731-1752, 2003.

19. van den Brand JM, Smits SL and Haagmans BL: Pathogenesis of Middle East respiratory syndrome coronavirus. J Pathol 235: 175-184, 2015.

20. Alsaad KO, Hajeer AH, Al Balwi M, Al Moaiqel M, Al Oudah N, Al Ajlan A, AlJohani S, Alsolamy S, Gmati GE, Balkhy H, et al: Histopathology of Middle East respiratory syndrome coronovirus (MERS-CoV) infection - clinico-pathological and ultrastructural study. Histopathology 72: 516-524, 2018.

21. Xu Z, Shi L, Wang Y, Zhang J, Huang L, Zhang C, Liu S, Zhao P, Liu H, Zhu L, et al: Pathological findings of COVID-19 associated with acute respiratory distress syndrome. Lancet Respir Med 8: 420-422, 2020.

22. Magro C, Mulvey JJ, Berlin D, Nuovo G, Salvatore S, Harp J, Baxter-Stoltzfus A and Laurence J: Complement associated microvascular injury and thrombosis in the pathogenesis of severe COVID-19 infection: A report of five cases. Transl Res 220: 1-13, 2020.

23. Wei PF; National Health Commission and National Administration of Traditional Chinese Medicine: Diagnosis and treatment of novel coronavirus pneumonia (Trial version 7). Chin Med J 133: 1087-1095, 2020.

24. Zhang MQ, Wang XH, Chen YL, Zhao KL, Cai YQ, An CL, Lin MG and Mu XD: Clinical features of 2019 novel coronavirus pneumonia in the early stage from a fever clinic in Beijing. Zhonghua Jie He He Hu Xi Za Zhi 43: 215-218, 2020 (In Chinese).

25. Guideline for medical imaging auxiliary diagnosis of coronavirus disease 2019. Zhongguo Yi Xue Ying Xiang Ji Shu 36: 321-330, 2020 (In Chinese).

26. Chinese Medical Association, Chinese Medical Doctor Association, China Research Hospital Association: Diagnostic guidelines for novel coronavirus pneumonia (2020 Version 1). New Med (Wars) 30: 22-34, 2020.

27. de Groot RJ, Baker SC, Baric RS, Brown CS, Drosten C, Enjuanes L, Fouchier RA, Galiano M, Gorbalenya AE, Memish ZA, et al: Middle East respiratory syndrome coronavirus (MERS-CoV): Announcement of the Coronavirus Study Group. J Virol 87: 7790-7792, 2013.

28. Zhu FQ and Lee G: Middle East respiratory syndrome. J Clin Inter Med 33: 91-93, 2016.

29. Meng JG and Lee YQ: Symptoms, imaging and laboratory diagnosis of Middle East respiratory syndrome. Zhonghua Linchuang Yishi Zazhi 43: 3-5, 2015.

30. Drosten C, Seilmaier M, Corman VM, Hartmann W, Scheible G, Sack S, Guggemos W, Kallies R, Muth D, Jun-glen S, et al: Clinical features and virological analysis of a case of Middle East respiratory syndrome coronavirus in-fection. Lancet Infect Dis 13: 745-751, 2013.

31. Ajlan AM, Ahyad RA, Jamjoom LG, Alharthy A and Madani TA: Middle East respiratory syndrome coronavirus (MERS-CoV) infection: Chest CT findings. AJR Am J Roentgenol 203: 782-787, 2014. 
32. Das KM, Lee EY, Enani MA, AlJawder SE, Singh R, Bashir S, Al-Nakshbandi N, AlDossari K and Larsson SG: CT correlation with outcomes in 15 patients with acute Middle East respiratory syndrome coronavirus. AJR Am J Roentgenol 204: 736-742, 2015.

33. Zhong NS: Diagnosis and treatment of SARS. Chin J Med 83: 95-116, 2003

34. Choi WJ, Lee KN, Kang EJ and Lee H: Middle East Respiratory Syndrome-Coronavirus Infection: A case report of serial computed tomographic findings in a young male patient. Korean J Radiol 17: 166-170, 2016.

35. Hijawi B, Abdallat M, Sayaydeh A, Alqasrawi S, Haddadin A, Jaarour N, Alsheikh S and Alsanouri T: Novel coronavirus infections in Jordan, April 2012: Epidemiological findings from a retrospective investigation. East Medi-terr Health J 19 (Suppl 1): S12-S18, 2013.

36. Chen $\mathrm{S}$ and Chen MQ: The recent progress on clinical control for Middle East respiratory syndrome. J Micro Infect 10: 208-214, 2015

37. WHO Mers-Cov Research Group: State of knowledge and data gaps of middle east respiratory syndrome coronavirus (MERS-CoV) in humans. PLoS Curr: Nov 12,2013 (Epub ahead of print). doi: 10.1371/currents.outbreaks.0bf719e352e7478f8ad8 5 fa30127ddb8.

38. Nowotny $\mathrm{N}$ and Kolodziejek J: Middle East respiratory syndrome coronavirus (MERS-CoV) in dromedary camels, Oman, 2013. Euro Surveill 19: 20781, 2014

39. Guan WJ, Ni ZY, Hu Y, Liang WH, Ou CQ, He JX, Liu L, Shan H, Lei CL, Hui DS, et al: Clinical Characteristics of Coronavirus Disease 2019 in China. N Engl J Med 382: 1708-1720, 2020.

40. Yang Y, Lu QB, Liu MJ, Wang YX, Zhang AR, Jalali N, Dean NE, Longini I, Halloran ME, Xu B, et al: Epidemiological and clinical features of the 2019 novel coronavirus outbreak in China. medRxiv: Feb 11, 2020 (Epub ahead of print). doi: org/10. 1101/2020.02.10.20021675.

41. Peiris JS, Chu CM, Cheng VC, Chan KS, Hung IF, Poon LL, Law KI, Tang BS, Hon TY, Chan CS, et al; HKU/UCH SARS Study Group: Clinical progression and viral load in a community outbreak of corona-virus-associated SARS pneumonia: A prospective study. Lancet 361: 1767-1772, 2003.

42. Lapinsky SE and Hawryluck L: ICU management of severe acute respiratory syndrome. Intensive Care Med 29: 870-875, 2003.

43. World Health Organization: State of knowledge and data gaps of Middle East respiratory syndrome coronavirus. Available from: http://www.who.int/csr/disease/coronavirus_infections/en.

44. Saad M, Omrani AS, Baig K, Bahloul A, Elzein F, Matin MA, Selim MA, Al Mutairi M, Al Nakhli D, Al Ai-daroos AY, et al: Clinical aspects and outcomes of 70 patients with Middle East respiratory syndrome coronavirus infection: A single-center experience in Saudi Arabia. Int J Infect Dis 29: 301-306, 2014

45. Lu PX, Yang GL, Yu WY, et al: Imaging follow-up of SARS patients complicated with pulmonary fibrosis. Chin J Med Imaging Tech 20: 1901-1903, 2004.

46. Kong Q and Qin C: Comparative study on pathogenesis of SARS pulmonary fibrosis. Chin J Comp Med 15: 335-338, 2005.

47. Lei J,Li J,Li X and Qi X: CT Imaging of the 2019 Novel Coronavirus (2019-nCoV) Pneumonia. Radiology 295: 18, 2020.

48. Vandenberg O, Martiny D, Rochas O, van Belkum A and Kozlakidis Z: Considerations for diagnostic COVID-19 tests. Nat Rev Microbiol 14: 1-13, 2020.

49. Hoque MN, Chaudhury A, Akanda MA, Hossain MA and Islam MT: Genomic diversity and evolution, diagno-sis, prevention, and therapeutics of the pandemic COVID-19 disease. PeerJ 8: e9689, 2020.

50. Shang L, Zhao J, Hu Y, Du R and Cao B: On the use of corticosteroids for 2019-nCoV pneumonia. Lancet 395: 683-684, 2020.

51. World Health Organization: Clinical management of severe acute respiratory infection when novel coronavirus $(\mathrm{nCoV})$ infection is suspected. Available from: https://www.who.int/ publications-detail/clinical-management-of-severe-acute-respiratoryinfection-when-novelcoronavirus-(ncov)-infection-is-suspected.

52. Du B, Qiu HB, Zhan X, Wang YS, Kang HY, Li XY, Wang F, Sun B and Tong ZH: Pharmacotherapeutics for the new coronavirus pneumonia. Zhonghua Jie He He Hu Xi Za Zhi 43 173-176, 2020 (In Chinese).
53. KooHJ,Lim S, Choe J, Choi SH, Sung H and Do KH: Radiographic and CT features of viral pneumonia. Radiographics 38: 719-739, 2018.

54. Guo YB: Clinical classification and early warning indicators of SARS. Chin Med News 18: 7-7, 2003.

55. Tongji Hospital Affiliated to Tongji Medical College of Huazhong University of Science and Technology, Peking Union Medical College Hospital, China-Japan Friendship Hospital: Diagnosis, treatment and management of the severe type of novel coronavirus pneumonia (Novel Coronavirus Pneumonia Treatment Cooperation Group of Tongji Hospital). J Inter Inten Med 26: 1-5, 2020.

56. Shi F, Yu Q, Huang W and Tan C: 2019 Novel Coronavirus (COVID-19) pneumonia with hemoptysis as the initial symptom: CT and clinical features. Korean J Radiol 21: 537-540, 2020.

57. Nitulescu GM,Paunescu H, Moschos SA, Petrakis D, Nitulescu G Ion GN, Spandidos DA, Nikolouzakis TK, Drakoulis N and Tsatsakis A: Comprehensive analysis of drugs to treat SARS CoV 2 infection: Mechanistic insights into current COVID 19 therapies (Review). Int J Mol Med 46: 467-488, 2020.

58. Calina D, Docea AO, Petrakis D, Egorov AM, Ishmukhametov AA Gabibov AG, Shtilman MI, KostoffR, Car-valho F, Vinceti M, et al: Towards effective COVID 19 vaccines: Updates, perspectives and challenges (Review). Int J Mol Med 46: 3-16, 2020.

59. World Health Organization: Coronavirus Disease 2020 (COVID-19) Pandemic. 16 September, 2020. Available from: https://www.who. int/news-room/q-a-detail/coronavirus-disease-(covid-19)-vaccines

60. Jones BM, Ma ES, Peiris JS, Wong PC, Ho JC, Lam B, Lai KN and Tsang KW: Prolonged disturbances of in vitro cytokine production in patients with severe acute respiratory syndrome (SARS) treated with ribavirin and steroids. Clin Exp Immunol 135: 467-473, 2004

61. Chien JY, Hsueh PR, Cheng WC, Yu CJ and Yang PC: Temporal changes in cytokine/chemokine profiles and pulmonary involvement in severe acute respiratory syndrome. Respirology 11 : 715-722, 2006.

62. Mahallawi WH, Khabour OF, Zhang Q, Makhdoum HM and Suliman BA: MERS-CoV infection in humans is associated with a pro-inflammatory Th1 and Th17 cytokine profile. Cytokine 104: 8-13, 2018

63. Liu J, Li S, Liu J, Liang B, Wang X, Wang H, Li W, Tong Q, Yi J, Zhao L, et al: Longitudinal characteristics of lymphocyte responses and cytokine profiles in the peripheral blood of SARS-CoV-2 infected patients. EBioMedicine 55: 102763, 2020.

64. Stern A, Skalsky K, Avni T, Carrara E, Leibovici L and Paul M Corticosteroids for pneumonia. Cochrane Data-base Syst Rev 12: CD007720, 2017.

65. Auyeung TW, Lee JS, Lai WK, Choi CH, Lee HK, Lee JS, Li PC, Lok KH, Ng YY and Wong WM: The use of corticosteroid as treatment in SARS was associated with adverse outcomes: A retrospective cohort study. J Infect 51: 98-102, 2005.

66. Lee N, Allen Chan KC, Hui DS, Ng EK, Wu A, Chiu RW, Wong VW, Chan PK, Wong KT, Wong E, et al: Effects of early corticosteroid treatment on plasma SARS-associated Coronavirus RNA concentrations in adult patients. J Clin Virol 31: 304-309, 2004.

67. Zhao JP, Hu Y, Du RH, Chen ZS, Jin Y, Zhou M, Zhang J, Qu JM and Cao B: Expert consensus on the use of corticosteroid in patients with 2019-nCoV pneumonia. Zhonghua Jie $\mathrm{He} \mathrm{He} \mathrm{Hu} \mathrm{Xi}_{\mathrm{i}}$ Za Zhi 43: 183-184, 2020 (In Chinese).

68. Fadel R, Morrison AR, Vahia A, Smith ZR, Chaudhry Z, Bhargava P, Miller J, Kenney RM, Alangaden G, Ramesh MS, et al: Early short-course corticosteroids in hospitalized patients with COVID-19. Clin Infect Dis 71: 2114-2120, 2020

69. Schoot TS, Kerckhoffs AP, Hilbrands LB and van Marum RJ: Immunosuppressive drugs and COVID-19: A Review. Front Pharmacol 11: 1333, 2020.

70. She Y: Is corticosteroid used or not used in the treatment of new coronavirus pneumonia? Chin J Crit Care Intensive Care Med 6: 53-55, 2020.

This work is licensed under a Creative Commons Attribution-NonCommercial-NoDerivatives 4.0 International (CC BY-NC-ND 4.0) License. 